\title{
Successful treatment with azacitidine for the simultaneous occurrence of multiple myeloma and acute myeloid leukemia with concomitant del(5q) and the $J A K 2$ V617F mutation
}

\author{
Satoko Oka ${ }^{1} \cdot$ Kazuo Ono $^{2} \cdot$ Masaharu Nohgawa ${ }^{1}$
}

Received: 17 May 2017 / Accepted: 23 May 2017 / Published online: 2 June 2017

(C) The Author(s) 2017. This article is an open access publication

Dear Editor,

A 73-year-old female with 5q-syndrome and the co-existent JAK2V617 mutation following 92-month treatment with lenalidomide exhibited evidence of disease progression, with leukopenia (WBC $2.2 \times 10^{9} / 1$ ), anemia (hemoglobin $68 \mathrm{~g} / 1$ ), and $30 \%$ blasts in her bone marrow (Fig. 1a). Strong nuclear p53 and CD34 immunostaining was detected in 30 and 10\% of hematopoietic cells, respectively (Fig. 1b), and CD138 immunostaining was noted in $10 \%$ of plasma cells (Fig. 1c). A FISH analysis revealed $5 \mathrm{q} 31$ in $86 \%$ of nuclei and molecular analyses were positive for the JAK2 V617F mutation (60\%). Her serum interleukin (IL)-6 level was $37 \mathrm{pg} / \mathrm{ml}$ (normal range, lower than $4 \mathrm{pg} / \mathrm{ml}$ ). The medullary plasma cell count was $<10 \%$. Serum IgG paraprotein and $\beta 2$-microglobulin $(\beta 2$ MG) levels increased to $65 \mathrm{~g} / \mathrm{l}$ (normal range, $7-16 \mathrm{~g} / \mathrm{l}$ ) and $7.8 \mathrm{mg} / \mathrm{l}$ (normal range $<2 \mathrm{mg} / \mathrm{l}$ ), respectively. The serum-free light chain ratio was 6.5 (normal range, 0.26-1.25). However, she had no evidence of myeloma-defining events or amyloidosis. A diagnosis of the simultaneous occurrence of acute myeloid leukemia (AML) and smoldering multiple myeloma (MM) was made, and 5 -azacytidine $\left(75 \mathrm{mg} / \mathrm{m}^{2}\right.$, days $1-7$ in a 28-day cycle) was initiated. After 2 cycles of this treatment, she had normal peripheral blood counts and no evidence of circulating blasts. Serum IgG paraprotein, $\beta 2$-microglobulin ( $\beta 2-\mathrm{MG}$ ) and IL-6 levels decreased to $18 \mathrm{~g} / 1,2.6 \mathrm{mg} / \mathrm{l}$, and $2.3 \mathrm{pg} / \mathrm{ml}$, respectively. A repeat marrow examination showed

Satoko Oka

okas@jasmine.ocn.ne.jp

1 Division of Hematology, Japanese Red Cross Society Wakayama Medical Center, Wakayama, Japan

2 Division of Pathology, Japanese Red Cross Society Wakayama Medical Center, Wakayama, Japan slightly hypercellular marrow, with a significant decrease in myeloblasts to $1 \%$ blasts and $5 \%$ of plasma cells (Fig. 1d). Strong nuclear p53 and CD34 immunostaining decreased in hematopoietic cells (Fig. 1e), and CD138 immunostaining was detected in a few plasma cells (Fig. 1f). Karyotypic analyses showed 46, XX, del(5q)(q13q31) (11/20 cells), 46, $\mathrm{XX}, \operatorname{del}(5 \mathrm{q})(\mathrm{q} 13 \mathrm{q} 31), \mathrm{t}(17 ; 21)(\mathrm{q} 25 ; \mathrm{q} 11.2)(9 / 20$ cells). A FISH analysis revealed $5 \mathrm{q} 31$ in $20 \%$ of nuclei and molecular analyses were positive for the JAK2 V617F mutation (8\%). The azacitidine treatment has been continued, and the patient maintained hematological $\mathrm{CR}$ without the progression of $\mathrm{MM}$ in the subsequent 12 months.

Concomitant occurrence of myelodysplastic syndrome (MDS)/myeloproliferative neoplasms (MPN), and MM is a rare event, and the reason of this association remains unclear. The management of these patients involves the treatment of MDS/MPN and the monitoring of MM for transformation to an overt plasma-cell malignancy. However, in patients who develop MM, management is focused on treating myeloma. Agents such as melphalan, thalidomide, lenalidomide, pomalidomide, and bortezomib exhibit clear activity in such patients and need to be considered in the treatment strategy. Azacitidine functions though the proteosomic destruction of DNA methyltransferase and resultant chromatin decondensation, and is not only active in higher-risk disease; similar response rates have been reported in IPSS low/int-1 patients including those with a del $(5 q)$ abnormality.

The frequencies of TP53 mutations in MDS and MM were $7-19$ and $8-15 \%$, respectively, and TP53 mutant clones may drive disease progression [1-4]. In MDS, p53 nuclear expression has been correlated with hemizygous TP53 mutations, and strong p53 immunostaining in $>1 \%$ of bone marrow progenitor cells has also been correlated with a higher risk of AML and resistance to lenalidomide therapy $[1,2,5]$. In MM, the presence of TP53 mutations indicates a dismal 

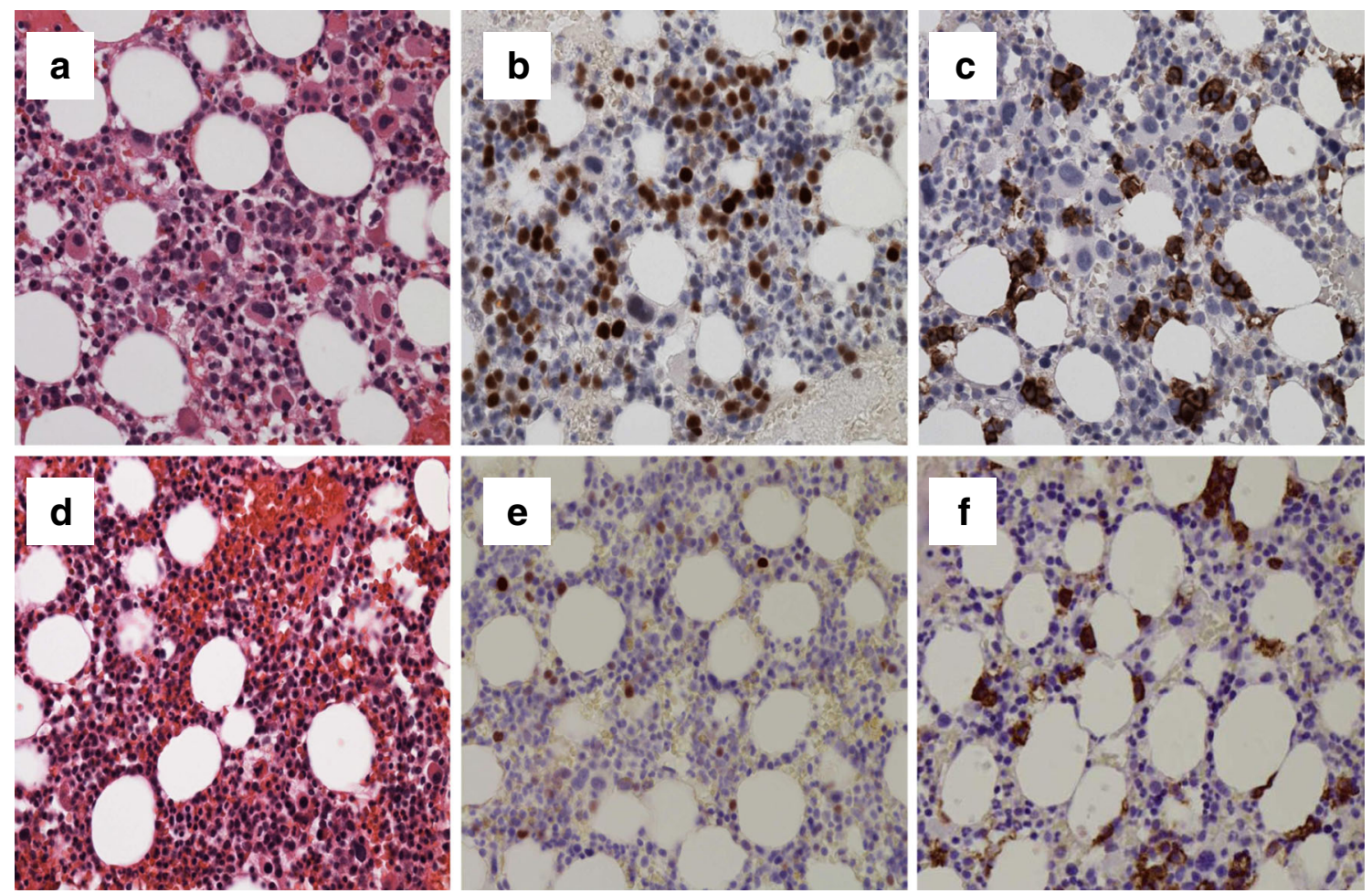

Fig. 1 Bone marrow biopsies of the concomitant occurrence of multiple myeloma and acute myeloid leukemia with $\operatorname{del}(5 \mathrm{q})$ and the JAK2 V617F mutation $(\mathbf{a}, \mathbf{b}, \mathbf{c})$ and after the azacitidine treatment $(\mathbf{d}, \mathbf{e}, \mathbf{f})$. a Hypercellular marrow with myelodysplasia-related changes and numerous blasts. b Strong nuclear p53 immunostaining in numerous

hematopoietic cells. c CD138 immunostaining in $10 \%$ of plasma cells. d Hypercellular marrow with myelodysplasia-related changes and decreased blasts. e Strong nuclear p53 immunostaining in a few hematopoietic cells. f CD138 immunostaining in a few plasma cells

prognosis, similar to MDS; patients exhibit a more aggressive disease course, more frequently have extramedullary disease and hypercalcemia, and have shorter overall and progressionfree survival $[3,4]$. In our case, the percentage of strongly p53-positive bone marrow cells was $30 \%$ at the time of the concomitant occurrence of leukemic transformation and MM; however, this percentage decreased after the treatment with azacitidine.

Another important distinction pertaining to the origin of these 2 clonal diseases is the involvement of the inflammatory cytokine, IL-6. IL-6 is a potent human myeloma-cell growth factor, and its overproduction is known to play a critical role as an anti-apoptosis-inducing agent in MM. IL-6 also promotes megakaryocytopoiesis and has been implicated in the pathogenesis of MPN [6]. The pathogenetic interactions between IL-6 and putative pluripotent stem cells may also be involved in the pathogenesis of co-existent cases of MM and MPN. Khong et al. recently demonstrated that azacitidine exerts pleiotropic effects including the downregulation of antiapoptotic factors (IL-6, IL-6 Receptor $\alpha$, and Bcl- ${ }^{-} \mathrm{LL}$ ) and JAK-STAT signaling as well as the inhibition of NFKB in MM cell lines [7]. Serum IL-6 levels increased in our case when SMM developed with the second relapse of AML, and decreased after the treatment with azacitidine.
In our case, azacitidine was effective for MDS transformed to AML and MM after the failure of a long-term treatment with lenalidomide. Although the pathogenetic interactions between IL-6, TP53 mutant clones, and putative pluripotent stem cells are not currently known, IL-6 and TP53 mutations may contribute to the relationship between MDS and MM. Further studies are warranted in order to improve clinical management and biological knowledge.

\section{Compliance with ethical standards}

Conflict of interest The authors declare that they have no conflict of interest.

Open Access This article is distributed under the terms of the Creative Commons Attribution 4.0 International License (http:// creativecommons.org/licenses/by/4.0/), which permits unrestricted use, distribution, and reproduction in any medium, provided you give appropriate credit to the original author(s) and the source, provide a link to the Creative Commons license, and indicate if changes were made.

\section{References}

1. Kita-Sasai $Y$, Horiike $S$, Misawa $S$ et al (2001) International prognostic scoring system and TP53 mutations are independent prognostic indicators for patients with myelodysplastic syndrome. Br J Haematol 115:309-312 
2. Mossner M, Jann JC, Nowak D et al (2016) Prevalence, clonal dynamics and clinical impact of TP53 mutations in patients with myelodysplastic syndrome with isolated deletion $(5 q)$ treated with lenalidomide: results from a prospective multicenter study of the German MDS study group (GMDS). Leukemia 30:1956-1959

3. Reece D, Song KW, Fu T et al (2009) Influence of cytogenetics in patients with relapsed or refractory multiple myeloma treated with lenalidomide plus dexamethasone: adverse effect of deletion $17 \mathrm{p} 13$. Blood 114:522-525

4. Dimopoulos MA, Kastritis E, Christoulas D et al (2010) Treatment of patients with relapsed/refractory multiple myeloma with lenalidomide and dexamethasone with or without bortezomib: prospective evaluation of the impact of cytogenetic abnormalities and of previous therapies. Leukemia 24:1769-1778

5. Saft L, Karimi M, Ghaderi M et al (2014) p53 protein expression independently predicts outcome in patients with lower-risk myelodysplastic syndromes with del(5q). Haematologica 99:10411049

6. Eskazan AE, Ongoren S, Ar MC, Soysal T, Ferhanoglu B, Aki H, Aydin Y (2011) Essential thrombocythemia and multiple myeloma: two rare diseases in one patient. Clin Lymphoma Myeloma Leuk 11: 442-445

7. Khong T, Sharkey J, Spencer A (2008) The effect of azacitidine on interleukin-6 signaling and nuclear factor-kappaB activation and its in vitro and in vivo activity against multiple myeloma. Haematologica 93:860-869 